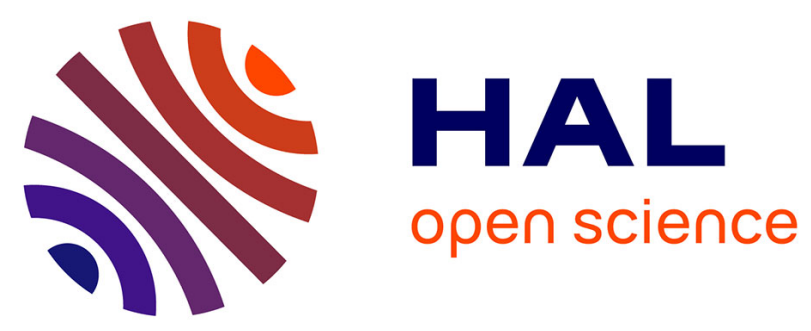

\title{
Nuclear fission time measurements as a function of excitation energy - A crystal blocking experiment
}

F. Barrué, S. Basnary, A. Chbihi, M. Chevallier, C. Cohen, D. Dauvergne, H. Ellmer, J.D. Frankland, D. Jacquet, R. Kirsch, et al.

\section{- To cite this version:}

F. Barrué, S. Basnary, A. Chbihi, M. Chevallier, C. Cohen, et al.. Nuclear fission time measurements as a function of excitation energy - A crystal blocking experiment. XIX International Conference on Atomic Collisions in Solids ICAS, Jul 2001, Paris, France. pp.852-859, 10.1016/S0168-583X(02)009151. in2p3-00012224

HAL Id: in2p3-00012224

https://hal.in2p3.fr/in2p3-00012224

Submitted on 29 Oct 2002

HAL is a multi-disciplinary open access archive for the deposit and dissemination of scientific research documents, whether they are published or not. The documents may come from teaching and research institutions in France or abroad, or from public or private research centers.
L'archive ouverte pluridisciplinaire $\mathbf{H A L}$, est destinée au dépôt et à la diffusion de documents scientifiques de niveau recherche, publiés ou non, émanant des établissements d'enseignement et de recherche français ou étrangers, des laboratoires publics ou privés. 


\title{
Nuclear fission time measurements as a function of excitation energy. A crystal blocking experiment
}

\author{
F.Barrué( $^{(1)}$, S.Basnary ${ }^{(2)}$, A.Chbihi ${ }^{(2)}$, M.Chevallier $^{(1)}$, C.Cohen $^{(3)}$, D.Dauvergne ${ }^{(1)}{ }^{*}, H$. \\ Ellmer $^{(3)}$, J.Frankland ${ }^{(2)}$, D.Jacquet ${ }^{(4)}$, R.Kirsch $^{(1)}$, P.Lautesse $^{(1)}$, A.L'Hoir $^{(3)}$, \\ M.Morjean $^{(2)}$, J-C.Poizat ${ }^{(1)}$, C.Ray ${ }^{(1)}$ and M.Toulemonde ${ }^{(5)}$
}

1) IPNL, CNRS/IN2P3, Université Lyon I, 3 rue Enrico Fermi, 69622 Villeurbanne cedex, France

2) GANIL, DSM/CEA, IN2P3/CNRS, BP 55027, 14076 Caen cedex, France

3) GPS, Universités Paris VI and Paris VII, 2 Place Jussieu, 75251 Paris cedex 05, France

4) IPNO, CNRS/IN2P3, Université Paris XI, BP 1, 91406 Orsay cedex, France

5) CIRIL, CNRS/CEA, rue Claude Bloch, 14040 Caen cedex, France

Abstract :

Fission times of lead and uranium nuclei have been measured at GANIL by the crystal blocking method. The inverse kinematics was used. Fragment atomic numbers and total excitation energies were determined. For data analysis, full MonteCarlo trajectory calculations were used to simulate the blocking patterns. The effect of post-scission emissions, included in our simulations, is discussed. At high excitation energies, the scissions occur dominantly at times shorter than $10^{-19} \mathrm{~s}$, whereas at low excitation energies $\left(E^{*}<250-300 \mathrm{MeV}\right)$, scissions occurring at much longer times with sizeable probabilities are observed both for uranium and for lead nuclei, leading to average scission times much longer than those inferred from pre-scission emission.

PACS numbers: $24.75 .+\mathrm{i}, 61.85 .+\mathrm{p}$

Keywords: Fission, Blocking

*Corresponding Author: d.dauvergne@ipnl.in2p3.fr 


\section{I - INTRODUCTION}

Measuring fission times of an excited nucleus provides information on nuclear dissipation[1]. During its deformation toward the saddle point (at which the fission process becomes irreversible), an excited nucleus cools down through particle evaporation (mainly neutron emission). During the cooling, the neutron binding energies as well as the fission barriers are modified at each evaporation step and, depending on the nuclei considered, the fission probability at very low residual excitation energies, after neutron evaporation, can either remain still sizeable or become negligible. Long lifetime components, associated with fission at low residual excitation energy, can thus show up for highly fissile nuclei. Therefore, the resulting statistical average fission times will strongly depend on the fission probability at low residual excitation energy. Moreover, damping of nucleon motion due to nuclear viscosity may slow down the whole evolution until the scission point is reached[2]. Thus the magnitude of the viscosity influences considerably the time needed by an excited nucleus to reach the scission point. The excited nucleus is thus a complex dynamical system. Its evolution depends on the interplay between cooling down and deformation, the velocity of which is limited by viscosity.

This shows the importance of measuring fission times for getting information on nuclear dissipation, and, in particular, long fission times $\left(>10^{-18} \mathrm{~s}\right)$ that cannot be predicted by the standard statistical theory[3] ignoring the effects of nuclear viscosity.

Various methods can be used to measure fission times. Pre-scission emission multiplicities (of particles as neutrons or Giant Dipole Resonance (GDR) $\gamma$-rays) can be related to fission times[4]. However, such relations suffer from two drawbacks: first, the evaluation of the fission time depends on the theoretical model used to describe the evaporation, and, second, pre-scission neutron and GDR $\gamma$-ray emission 
probabilities become very small at low residual excitation energies, which makes this method very little sensitive to the long times that are associated to low residual excitation energies. The crystal blocking technique, as used in this work, is certainly more straightforward because it measures in a model-independent way the recoil distance covered by the excited nucleus during the whole fission process (starting from the initial collision and ending at the scission point).

\section{II - FISSION TIME MEASUREMENTS BY CRYSTAL BLOCKING.}

This application of the blocking technique was proposed very soon after channeling effects were observed to affect the propagation of charged particles in aligned crystals[5-7]. During the collision of a projectile with a crystal atom, a nucleus is given an excitation energy $\mathrm{E}^{\star}$ and a recoil momentum $M \vec{v}$. Then the fission occurs at a recoil position $\vec{r}_{f}=\vec{v} t_{f}$, where $t_{f}$ is the scission time. At this stage the continuum potential approximation is useful to understand the blocking effect. In this approximation[8], the target atomic potential is averaged along the crystallographic direction of interest (axis or plane). In the following we will focus on axial effects. The transverse motion of a particle is separated from its longitudinal one. Individual collisions with the target atoms are ignored, as well as multiple scattering on electrons. At the fission location $\vec{r}_{f}$ a fission fragment is given a transverse energy

$$
E_{\perp}=q_{f} U\left(\vec{r}_{f \perp}\right)+E_{f} \Psi_{f}^{2}
$$

where $q_{f}, E_{f}, \Psi_{f}$ are respectively the fragment charge, kinetic energy and emission angle relative to the atomic string in the laboratory frame. $U\left(\vec{r}_{\perp}\right)$ is the continuum potential of the string, at a position $\vec{r}_{\perp}$ in the transverse plane defined as 
the projection of the recoil vector $\vec{r}$ in this plane. $E_{\perp}$ is the sum of a potential term and a kinetic term. If one neglects energy loss and charge exchange, and uses the hypotheses mentioned above, $E_{\perp}$ is conserved throughout the fragment path in the crystal. Within this approximation, the condition for the fragment to emerge from the crystal at an angle $\theta$ smaller than the critical channeling angle $\Psi_{c}=\sqrt{q_{f} U_{\max } / E_{f}}$ with respect to the axial direction is

$$
E_{\perp} \leq q_{f} U_{\max }
$$

where $U_{\max }$ is the maximum value of the transverse potential. $U_{\max }$ is reached at distances from the strings closer than $u_{2}$, that characterizes the thermal vibrations of the lattice atoms. For very short $t_{f}$ values, $U\left(\vec{r}_{f \perp}\right) \cup U_{\max }$ and most of the fragments are deflected at angles greater than $\Psi_{c}$. The blocking effect is weaker if the fission fragment has a smaller transverse energy, i.e. if it is emitted at a larger distance from the string.

One can then define the time sensitivity range of the blocking method for fission time measurements. Consider the probability $d N / d \Omega\left(\theta \leq \Psi_{c}\right)$ for a fragment to emerge from the crystal at an angle smaller than $\Psi_{c}$ with respect to the axial direction. In the time sensitivity range $d N / d \Omega\left(\theta \leq \Psi_{c}\right)$ increases with the mean fission time $\tau_{f}$, i.e. the corresponding $U\left(r_{\perp}\right)$ is a decreasing function of $r_{\perp}\left(\tau_{f}\right)$.

- For short times corresponding to transverse recoils $r_{f \perp}=v_{\perp} t_{f}$ smaller than $u_{2}$, the blocking effect is maximum. Such times, smaller than ${ }_{\min }=u_{2} / v_{\perp}$, are out of the time sensitivity range and lead to the same result as infinitely short times. 
- On the other hand, the upper time limit $t_{\max }$ corresponds to transverse recoil distances typically larger than the lattice constant $d_{\text {lattice }}$. Fissions occurring at times longer than $t_{\max }$ will take place at positions where the transverse potential ranges randomly between 0 and $U_{\max }$. This leads to a uniform angular distribution $d N / d \Omega(\theta)$ at the crystal exit, because blocking effects are compensated by channeling effects (although Andersen et al. pointed out that, given a recoil direction, there are fission time distributions that might give rise to a flux peaking at $\theta=0$ [9]).

For scission time distributions ranging from $t_{f}<t_{\min }$ to $t_{f}>t_{\max }$, blocking experiments can provide the relative fractions of short and long time components. However, when the scission time distribution is broad (of the order of the sensitivity range of the blocking technique or beyond), it becomes very difficult, due to the finite statistics available in any experiment, to extract univocally from the data the actual time distribution. The evaluation of mean fission times will depend essentially on the assumed longest scission times.

This is illustrated in fig.1, where blocking dips around the $<110>$ axis of silicon are simulated for various time distributions in the case of the symmetrical fission of ${ }^{208} \mathrm{~Pb}$ (for $29 \mathrm{MeV} / \mathrm{u}$ incident $\mathrm{Pb}$ ions, corresponding to one of the experimental situations described below). In this case, where the inverse kinematics is used, the time sensitivity window is found to range roughly between $3.10^{-19} \mathrm{~s}$ and $10^{-16} \mathrm{~s}$ for exponential fission time distributions. Single exponential time distributions for the fission of an excited nucleus are certainly not realistic due to the complexity of this process, as mentioned in the introduction. We used another purely arbitrary type of time distribution to simulate another blocking dip shown in fig.1. We used a twocomponent distribution with one at very short times (mean value $\tau_{f}=10^{-19} \mathrm{~S}<t_{\min }$, 
the shape of this distribution being of no importance), and a second one being uniformly distributed between 0 and $6.10^{-16} \mathrm{~s}$ (providing a uniform distribution of $r_{f \perp}$ values). The relative weights of these two components are $97 \%$ and $3 \%$, respectively, corresponding to an average time $\tau_{f}=10^{-17} \mathrm{~s}$. This time distribution may not be realistic either, although the longer time limit is of the order of the values obtained by Forster et al., in blocking experiments for the fission of lead-like nuclei with a sensitivity window shifted towards longer times[10] (due to the direct kinematics used in their case). The simulation performed with this two-component distribution shows that small fractions of long fission times may be evidenced experimentally by the blocking technique. This last simulated dip is nearly equal to the weighted sum of the dips obtained for $\tau_{f}<t_{\min }$ and for the uniform distribution corresponding to $\tau_{f}>t_{\max }$, respectively. The main difference with the distribution associated to $\tau_{f}<t_{\min }$ is the enhancement of the minimum of $d N / d \Omega(\theta)$ for $\theta \cup 0$. The difference between this dip and the one corresponding to a simple exponential distribution with same mean fission time $\tau_{f}=10^{-17} \mathrm{~s}$ is striking.

So far we have described the principle of fission time measurements by blocking in terms of angular deflections caused by the continuum transverse potential at the place where scission occurs inside the crystal. Actually, a few points have to be discussed in order to go beyond this simple picture.

- First of all, the continuum potential model cannot lead to a correct description of fragment trajectories close to the atomic strings. Individual atomic collisions have to be calculated in the simulations to account for elastic scattering by target nuclei. - Also, fission fragment blocking patterns may be influenced by the effects of electronic multiple scattering, energy loss and charge exchange, that cause the non- 
conservation of transverse energy. As we will see below, partial information on such effects can be obtained experimentally through blocking studies of a "zero lifetime" process, like Rutherford scattering.

- After scission, the fission fragments are left in excited states. They will dissipate their excitation energy by emitting $\gamma$-rays or light particles like neutrons or $\alpha$ particles. For each post-scission emission, the maximum angular deflection $\delta \theta_{\max }$ of the fragment is given by the ratio of the evaporated particle momentum $p_{\text {post }}$ in the center of mass frame to the fragment momentum $p_{f}$ in the laboratory frame: $\delta \theta_{\text {max }} \cup p_{\text {post }} / p_{f}$. This deflection angle has to be compared to the characteristic magnitude of angular deflections caused by the blocking, i.e. the channeling critical angle $\Psi_{c}$. The influence of this effect on the blocking dip is then connected to the ratio

$$
\frac{\delta \theta_{\max }}{\Psi_{c}} \cup \frac{p_{\text {post }}}{\sqrt{\frac{4 Z_{2} M_{f} q_{f} e^{2}}{d}}}
$$

where $Z_{2}$ and $d$ are the crystal atomic number and inter-atomic distance along the string, respectively, $M_{f}$ and $q_{f}$ are the fragment mass and charge. Thus, the relative perturbation of blocking effects by a single post-scission emission is seen to be independent of the fragment energy.

This effect of delayed particle emission by an excited nucleus after inelastic collisions has been previously studied for the blocking of light ions[11,12,13]. For heavy fission fragments, the emission of low momentum particles like $\gamma$-rays can generally be neglected. On the contrary, post-scission neutron evaporation can lead to noticeable modifications of the blocking dips. Nevertheless, the main problem for 
evaluating post-scission emission effects is the lack of accurate knowledge of the time and energy distribution for post-scission neutrons, and in particular when the fragments reach very low residual excitation energies. However, significant effects can be predicted for fragments formed after fast fissions $\left(\tau_{f} \leq t_{\text {min }}\right)$; these effects are associated to long particle emission times. In such cases the fragments are emitted with a transverse energy $E_{\perp} ? q_{f} U_{\max }$. At the place where evaporation occurs, the potential term may be small, the recoil associated to the particle evaporation can then significantly lower the kinetic term, allowing the transverse energy of some fragments to fall below the critical transverse energy for blocking: the fragments cool down in the transverse space.

\section{III - EXPERIMENTS}

We have studied fission times of uranium and lead as a function of excitation energy. For the first nucleus, that has a low fission barrier, the fission probability is sizeable whatever the excitation energy is. The existence of long fission time components had been already observed at low excitation energies in blocking experiments[14,15] and experiments using the time scale for $\mathrm{x}$-ray filling of K-shell vacancies[16] whereas pre-scission neutron multiplicities[17] or GDR- $\gamma$-rays[18] provided only times shorter than $10^{-18} \mathrm{~s}$.

For lead nuclei, the fission at very high excitation energy is expected to be fast, like for uranium nuclei. The fission barrier is higher in the case of lead. Short fission times are also expected at low excitation energies, because only first chance fissions are allowed, as explained in the introduction. Longer fission times could be expected at intermediate excitation energies, where fission could still occur after several neutron emissions. Previous blocking experiments $[9,10,19]$ have reported very high 
yields $d N / d \Omega(\theta \cup 0)$ of fission fragments observed close to axial directions, for fusionfission of compound nuclei with $A \leq 200$, and excitation energies of the order of 100 MeV. These high yields were attributed to fission time components extending to times longer than $10^{-16} \mathrm{~s}$ (according to the time sensitivity window of these experiments).

The experimental setup as well as the results of our uranium experiment have been described in refs. [20,21]. Briefly, $24 \mathrm{MeV} / \mathrm{u}^{238} \mathrm{U}$ ions (respectively $29 \mathrm{MeV} / \mathrm{u}$ ${ }^{208} \mathrm{~Pb}$ ions) were sent onto a $6 \mu \mathrm{m}$ (respectively $15 \mu \mathrm{m}$ ) thick silicon crystal. Both fission fragments were detected in coincidence and Z-identified using $E-\Delta E$ telescopes. The telescopes devoted to blocking were $\mathrm{X}$ and $\mathrm{Y}$ position sensitive, and located $3 \mathrm{~m}$ (respectively $3.5 \mathrm{~m}$ ) behind the crystal. The blocking patterns of fission fragments were observed with the $<110>$ axis of silicon directed to the position sensitive telescope located at $7^{\circ}$ (respectively $5^{\circ}$ ) with respect to the incident beam direction. The use of small forward angles for fission blocking observation is imposed by the inverse kinematics, which reduces the advantage of large fissioning nuclei velocities. However, the recoil velocity - and in particular the transverse component is nearly the same for all excited nuclei (almost independent on the excitation energy and not sensitive to perturbations caused by pre-scission evaporations). This makes our configuration sensitive to shorter times than other blocking experiments using low energy light projectiles on heavy targets. Moreover, the use of swift fragments and of thin-, low Z- crystals minimizes the effect of angular multiple scattering.

The total excitation energy, ranging from 0 to $600 \mathrm{MeV}$ (respectively 0 to 800 $\mathrm{MeV}$ ) was measured for each fission event by the $4 \pi$ ORION neutron detector. In the experiment using the lead ion beam, the beam intensity was $10^{9}$ particle/s, two orders of magnitude higher than during the experiment with uranium ions, a counting 
rate too high to allow direct determination of neutron multiplicities. Thus we used the "prompt" signal from this detector, arising mainly from the energy loss of neutrons in the scintillator, and therefore correlated to the excitation energy [22].

Possible damaging of the irradiated crystal was controlled by measuring the quality of blocking for elastic scattering at $1^{\circ}$ from the primary beam. No significant damage was observed after impact of more than $10^{12}$ uranium ions on a spot of about $1 \mathrm{~mm}$ diameter. For the lead experiment, the beam impact was changed periodically to keep the fluence at values below $10^{13}$ ions $/ \mathrm{mm}^{2}$. Elastic blocking patterns were recorded before and after each impact irradiation. Off-line analysis allowed us to check that crystal damaging was negligible.

\section{SIMULATIONS AND RESULTS}

Simulations using full Monte-Carlo trajectory calculations are necessary to reproduce and interpret blocking dips. Each individual elastic collision with target atoms were calculated within the Moliere approximation of Thomas-Fermi potentials. Experimental factors that could lead to a filling of blocking patterns were taken into account empirically: such factors are the position resolution of the detector, the beam spot size, the electronic multiple scattering in the target and, possibly, crystal defects. To account for these factors we considered the blocking dips of projectiles elastically scattered at $1^{\circ}$ from the beam. Such a typical dip is presented in Fig. 2 for lead projectiles, together with the corresponding simulation. In order to reproduce the experimental dip, the simulated angular distribution corresponding to an "ideal" experiment has been convoluted with a Gaussian distribution with $\sigma=0.009^{\circ}$, which shows that the dominant factor in this spread out is the beam spot shape. The same experimental factor has been used for all further fission fragment blocking pattern 
simulations. Hence we neglect possible (but minor) different influences due to different multiple scattering between fission fragments and elastically scattered incident ions.

Also charge exchange may modify the transverse energy of ions in the crystal. In particular, the charge state of an ion undergoing close collisions with the atomic string just after the violent nuclear collision may change during its path in the crystal. In our simulations we neglect charge exchange and use the mean charge state at equilibrium throughout the crystal. We consider that fragments are point-like charges.

The blocking dips obtained for the fission of uranium nuclei have been presented in figs. 2 and 3 of ref. [21] as a function of excitation energy (with the condition $Z_{f 1}+Z_{f 2}=92 \pm 1$ for the detected fragments). The time distributions used to fit these dips are made up of two components: one corresponding to very short times $\left(\tau_{f} \cup 10^{-19} \mathrm{~s}<t_{\min }\right)$ and a long time component (uniform distribution ranging from 0 to $6.10^{-17} \mathrm{~s}$ ). Post-scission neutron emissions were included in the simulations according to the mean values for emission times and energies from a statistical code[23].

The relative weights $X_{\text {long }}$ of the long time components decrease with excitation energy, with values $X_{\text {long }} ? 40 \%, X_{\text {long }} \cup 20 \%, 10 \%, 6 \%$ and $0 \%$, corresponding to ranges of excitation energy with average values $E^{*} \leq 20 \mathrm{MeV}, E^{*} \cup 60 \mathrm{MeV}, 120$ $\mathrm{MeV}, 160 \mathrm{MeV}$ and $250 \mathrm{MeV}$, respectively. Such long fission time fractions are compatible with those measured in refs. $[14,15]$ at smaller excitation energies. Average fission times deduced in [21] from the long fission time fractions have been used by Gontchar et al.[24] to adjust phenomenologically the wall term of the walland-window formula for one-body dissipation in the CDSM2 model. 
In the same way, we have selected excitation energies for the fission of lead $\left(Z_{f 1}+Z_{f 2}=82\right.$, with an uncertainty of one charge unit). Blocking dips corresponding to high $\left(E^{*}>400 \mathrm{MeV}\right)$ and low $\left(E^{*}<300 \mathrm{MeV}\right)$ excitation energies are presented in Fig.3-a and b respectively. They are associated to fragments emitted in the forward direction in the center of mass frame. In fig.3-a, the high excitation energy blocking dip is presented together with the results of simulations using a mean fission time $\tau_{f} \leq 10^{-19} \mathrm{~S}$ (presented as a dashed curve). The bottom of the experimental dip is significantly higher than what is predicted by this simulation. This could suggest the existence of longer fission times. However, such a long fission time fraction should decrease when $E^{*}$ increases, a behavior that is not observed in the data (subdivision in energy ranges between 300 and $800 \mathrm{MeV}$ provides identical dips). Actually, this poor agreement has been obtained with a simulation in which the effects of post-scission emission have been neglected, although in the case of "fast" fission, the fission fragments are left after scission with rather high excitation energies. We have therefore included this effect in our simulations, considering neutron evaporation by initial fragments with $Z_{f}=41, A=104$ and $E^{*}=100 \mathrm{MeV}$. The characteristics of the neutrons (energy and emission time) have been calculated with the statistical code SIMDEC[25]. As discussed in section II, only neutrons emitted at sufficiently long time after fission $\left(t>10^{-18} \mathrm{~s}\right.$, i.e. at low residual excitation energies) have a significant influence on the blocking dips. This is indeed confirmed in our Monte-Carlo simulations. However, the neutron emission times are strongly dependent on the level density parameter value assumed in SIMDEC. In order to get the most reliable behavior at long emission time, we used the value inferred from neutron resonance studies[26] at low excitation energies for nuclear masses around $A=100$. As shown in fig. 3 -a, a significantly better agreement with the data is 
reached. Considering the rough assumptions performed on the fission fragment mass, charge, and energy, and the difficulty to adjust the statistical model parameters for post-scission emission, such an agreement can be considered as quite satisfactory, particularly in the minimum yield.

Similar simulations to the ones that fit satisfactorily the experimental dip in fig.3a $\left(E^{*}>400 \mathrm{MeV}\right)$ are compared, in fig.3-b (dotted line), to the experimental dip corresponding to low excitation energies $\left(E^{*}<300 \mathrm{MeV}\right)$. A significant difference is observed. As the effect of post-scission neutron evaporation is already included in the simulations, the discrepancy, in this case, can only be attributed to a contribution of long fission times that did not show up at high excitation energies. We have therefore introduced a fraction $X_{\text {long }}$ of fission events occurring at times ranging uniformly from 0 to $10^{-16} \mathrm{~s}$. As shown by the full curve in fig.3-b, a better agreement is obtained, especially for the bottom of the dip, with $X_{\text {long }}=7 \pm 3 \%$.

New information will be extracted from the present experiment after further analysis. For instance, fission occurring at lower excitation energies (after emission of an $\alpha$-particle) will be studied separately. Also planar blocking effects can be used to study very long fission times (above $10^{-16} \mathrm{~s}$ ), because the crystal orientation was chosen in such a way that the time needed by the excited nuclei to recoil from the (101) plane $\left(t_{\min }=2 \leftrightarrow 0^{-17} \mathrm{~s}\right)$ was more than one order of magnitude longer than for the $\langle 110\rangle$ axis. This will be used to improve the scission time distribution introduced in our simulations to extract the fraction $\mathrm{X}_{\text {long. }}$.

The existence of long fission times for $E^{*}<300 \mathrm{MeV}$ observed in the present experiment seems to be in agreement with the conclusions of previous blocking experiment for the fission of lead nuclei with $A \leq 200[9,10,19]$ (according to Sierk systematics, the fission barriers (around $13 \mathrm{MeV}$ ) decrease by less than $1 \mathrm{MeV}$ 
between $A=207$ and $A=200$; therefore the different lead isotopes considered in the various blocking experiments should only take into account weak discrepancies). Nevertheless, although these experiments were less sensitive to post-scission emissions (due to the use of high- $Z$ crystals), the long fission time components that were deduced from these experiments were probably overestimated, because postscission emission was neglected in their analysis. However, all the experiments performed up to now in order to reach pieces of information on the fission time scales involved in the fission process of lead nuclei have shown the existence of long fission times with sizeable weights, corresponding to average scission times longer than 10-

${ }^{19} \mathrm{~S}$. Like in the case of uranium[21], a strong discrepancy seems to exist between these long average times and the much shorter times inferred from pre-scission emission[4].

\section{V - SUMMARY}

We have measured fission times by the blocking technique in single crystals over a wide range of excitation energies for two very different nuclei. For highly fissile uranium nuclei, a large fraction of long fission times (above $10^{-17} \mathrm{~s}$ ) is observed at low excitation energy, and vanishes progressively above $200 \mathrm{MeV}$. Such long times are in agreement with previous blocking measurements at low excitation energies, and they provide a new piece of information on nuclear dissipation. For the much less fissile lead nuclei, the evolution of the blocking dips has been analyzed for excitation energies larger than $400 \mathrm{MeV}$ and lower than $300 \mathrm{MeV}$. The blocking dip measured for the highest excitation energy is reproduced by simulations considering only short fission times $\left(\tau_{f} \leq 10^{-19} \mathrm{~s}\right)$, provided the huge effect of post-scission emission is taken into account. For the lowest excitation energies, a significant fraction of long fission 
times is observed, leading to an average fission time longer than $10^{-19} \mathrm{~s}$. Like in the case of uranium, the fission times inferred by the blocking technique seem much longer than those inferred from pre-scission emission.

\section{REFERENCES:}

[1] D. Hilscher and H. Rossner, Ann. Phys. Fr. 17, 471 (1992)

[2] Yu. A. Lazarev et al., Phys. Rev. Lett. 70, 1220 (1993)

[3] N. Bohr and J.A. Wheeler, Phys. Rev. 36, 426 (1939)

[4] D. J. Hinde, Nucl. Phys. A553, 255c (1993)

[5] A. F. Tulinov, Dokl. Akad. Nauk. SSSR 165, 546 (1965) [Sov. Phys.-Doklady 10, 000 (1966)].

[6] D. S. Gemmell and R.E. Holland, Phys. Rev. Lett. 14, 945 (1965).

[7] W. M. Gibson, Ann. Rev. Nucl. Sci. 25, 465 (1975).

[8] J. Lindhard, K. Dan. Vidensk. Selsk. Mat. Fys. Medd. 34 (1965) n¹4.

[9] J. U. Andersen et al., K. Dan. Vidensk. Selsk. Mat. Fys. Medd. 40 (1980) n7.

[10] J. S.Forster, I. V. Mitchell, J. U. Andersen, A. S. Jensen, E. Laegsgaard, W. M. Gibson and K. Reichelt, Nucl. Phys. A464, 497 (1987).

[11] R. F. A. Hoernlé, R. W. Fearick and J. P. F. Sellschop, Phys. Rev. Lett. 68, 500 (1992).

[12] F. Malaguti, G. Giardina and P. Olivo, Nucl. Instr. And Meth. B129, 341 (1997).

[13] S.A. Karamyan, Nucl. Instr. And Meth. B51, 354 (1990).

[14] J. U. Andersen, K. O. Nielsen, J. Skak-Nielsen, R. Hellborg and K. G. Prasad, Nucl. Phys. A241, 317 (1975).

[15] Yu. V. Melikov, Yu. D. Otstavnov, A. F. Tulinov and N. G. Chetchenin, Nucl. Phys. A180, 241 (1972). 
[16] J. D. Molitoris et al., Phys. Rev. Lett. 70, 537 (1993).

[17] D. J. Hinde, D. Hilscher and H. Rossner, Nucl. Phys. A502, 497c (1989).

[18] R. Butsch, D. J. Hofman, C. P. Montoya, P. Paul and M. Thoennessen, Phys.

Rev. C44, 1515 (1991).

[19] V. V. Kamanin, S. A. Karamyan, F. Normuratov and S. P. Tret'Yakova, Sov. J.

Nucl. Phys. 16, 249 (1973) [Yad. Fyz. 16, 447 (1972)].

[20] M. Morjean et al., Nucl. Phys. A630, 200c (1998).

[21] F. Goldenbaum et al., Phys. Rev. Lett. 82, 5012 (2000).

[22] M. Morjean et al., Nucl. Phys. A591, 371 (1995)

[23] R. J. Charity et al., Nucl. Phys. A483, 371 (1988).

[24] I. Gontchar, M. Morjean and S. Basnary, GANIL P0018 (2000)

[25] M. Ohta et al., Proc. of Tours Symposium on Nuclear Physics II, World Scientific (1994) 480.

[26] J. R. Huizenga and L. G. Moretto, Ann. Rev. Nucl. Sci. 22, 427 (1972). 


\section{FIGURE CAPTIONS:}

Figure 1:

Simulation of blocking dips for $Z=41$ fission fragments of incident lead nuclei. The crystal axis is the $<110>$ axis of silicon, oriented at $5^{\circ}$ from the incident $29 \mathrm{MeV} / \mathrm{u}$

${ }^{208} \mathrm{~Pb}$ beam. Various fission time distributions are used. All dips represented by solid lines correspond to simple exponential distributions. The dashed-line dip is obtained using a two-component time distribution (see text).

Figure 2:

Experimental blocking dip around the $<110>$ axis of silicon for elastic scattering at $1^{\circ}$ from the incident $\mathrm{Pb}$ ion beam at $29 \mathrm{MeV} / \mathrm{u}$. The line is the adjusted result of the simulations.

Figure 3:

Experimental blocking dips for the fission of lead-like nuclei $\left(Z_{f 1}+Z_{f 2}=82\right)$. The selected fragments were emitted in the forward direction in the center of mass.

(a) $E^{*}>400 \mathrm{MeV}$. Dashed curve: simulation using $Z_{1}=41$, average fission time $\left\langle\tau_{f}\right\rangle \leq 10^{-19} \mathrm{~s}$, without post-scission emission. Solid line: same simulation including post-scission emission.

(b) $E^{*}<300 \mathrm{MeV}$. Dotted curve: simulation with average fission time $\left\langle\tau_{f}\right\rangle \leq 10^{-19} \mathrm{~s}$, including post-scission emission. Solid line: Simulation using 93\% of short fission times as above, and $7 \%$ of fission times ranging uniformly between 0 and $10^{-16} \mathrm{~s}$. 


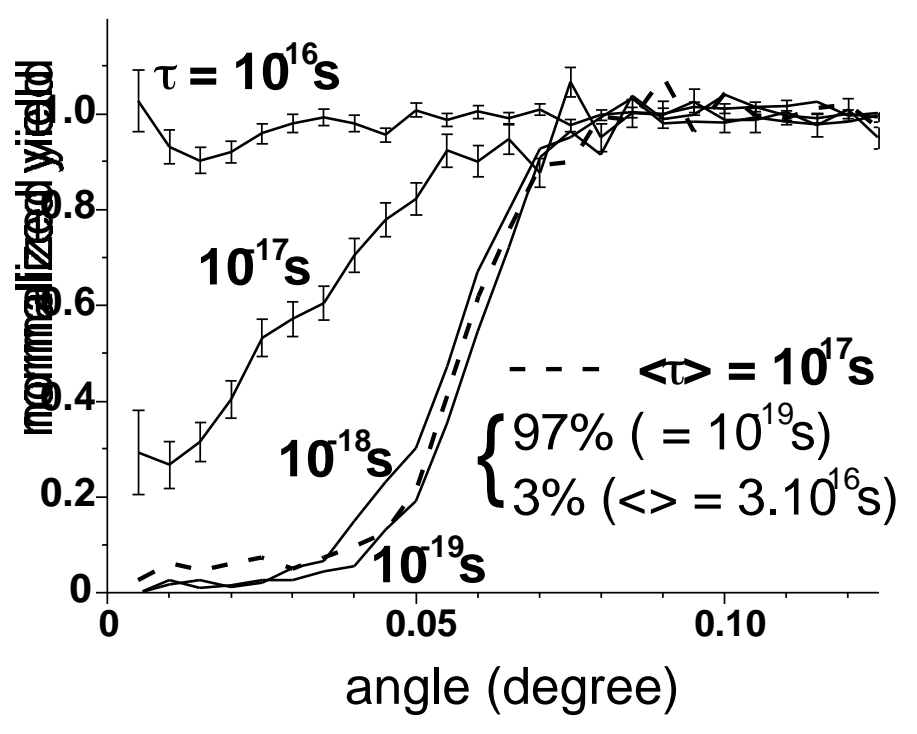

Figure 1

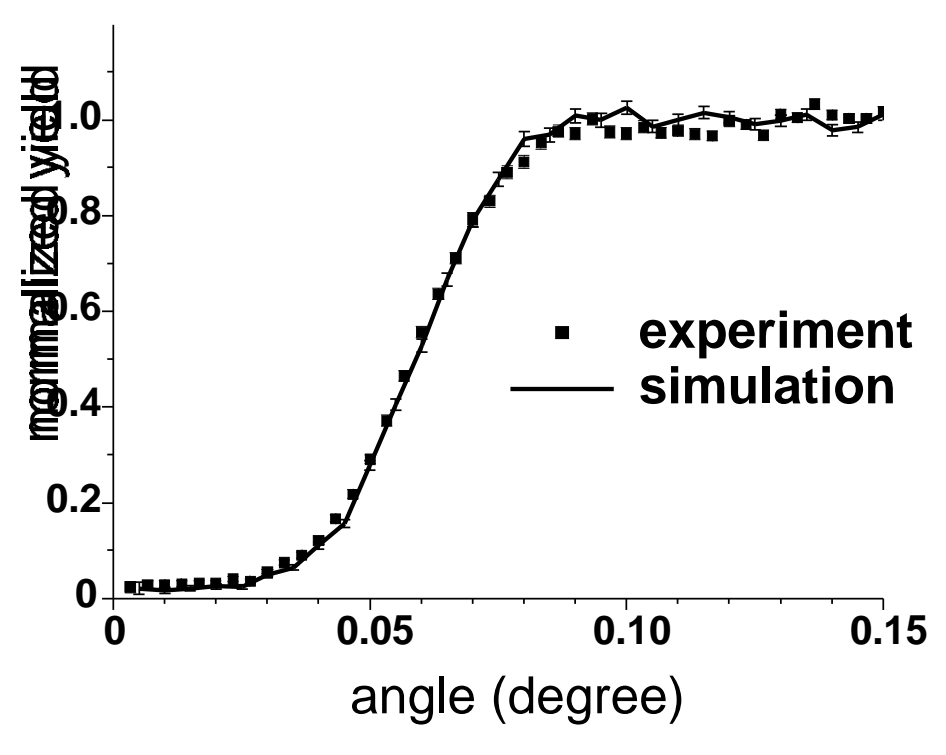

Figure 2 


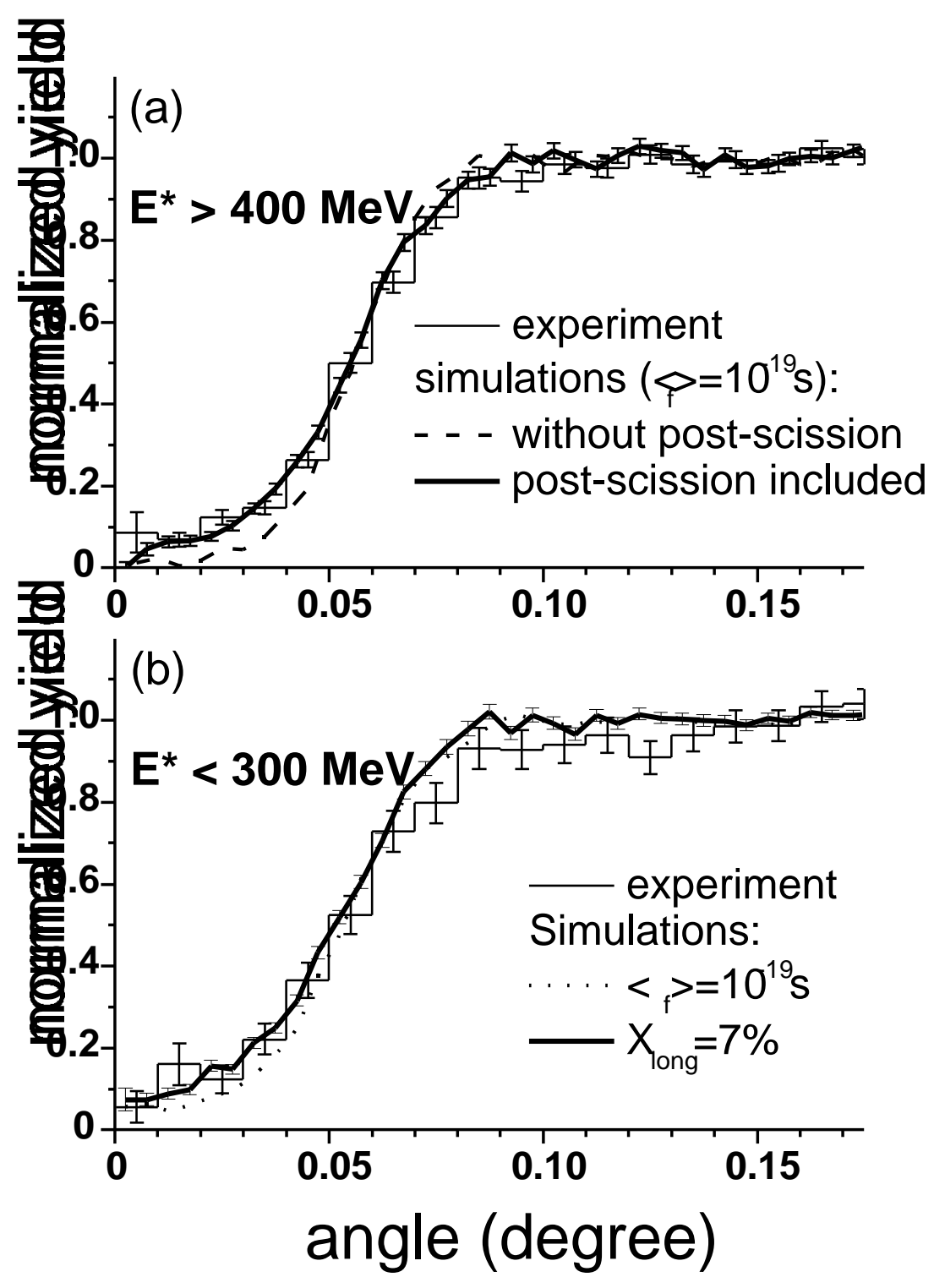

Figure 3 